\title{
Pulmonary involvement in connective tissue disease due to Coronavirus 19: a case report
}

\author{
๑Ömer Faruk Topaloğlu, @Halil Özer, (1)Abidin Kılınçer, @Emine Uysal \\ Selcuk University, Faculty of Medicine, Department of Radiology, Konya, Turkey
}

Cite this article as: Topaloğlu ÖF, Özer H, Kılınçer A, Uysal E. Pulmonary involvement in connective tissue disease due to Coronavirus 19: a case report. J Health Sci Med 2021; 4(3): 394-396.

\begin{abstract}
Coronavirus disease (COVID-19) is a disease caused by the new coronavirus called severe acute respiratory syndrome coronavirus 2 (SARS-CoV-2) emerging in Wuhan, China's Hubei province. The epidemic spread exponentially across the world and became a pandemic. Real-time fluorescence reverse transcription- polymerase chain reaction (RT-PCR) is the preferred initial and reference diagnostic test for COVID-19. Chest computed tomography (CT) has gained importance due to the long result time and false negativity of the RT-PCR test. Due to immune dysfunction, steroid usage and immunotherapy, connective tissue diseases are vulnerable to viral infections. In this article, we aimed to present the chest CT findings of a patient with connective tissue diseases who received immunosuppressed therapy. A 28-year-old female patient applied to COVID-19 outpatient clinic with complaints of fever and weakness. The patient had a history of Sjogren's syndrome, mixed connective tissue disease and autoimmune hepatitis. In the chest computed tomography taken after the first examination, blood analysis and chest radiography, the patient had signs of interstitial lung disease, and a round-shaped peripheral ground glass opacity was observed in the lower lobe of the right lung, which was understood to be newly developed according to previous examinations. These findings suggested COVID-19 pneumonia in addition to interstitial lung disease and the RTPCR test confirmed COVID-19. Concomitant chronic diseases, especially diseases affecting the immune system, increase the risk for COVID-19 as in other infective diseases
\end{abstract}

Keywords: Connective tissue disease, Sjogren's syndrome, pneumonia, COVID-19, ground glass opacity

\section{INTRODUCTION}

Coronavirus disease (COVID-19) is a disease caused by the new coronavirus called severe acute respiratory syndrome coronavirus 2 (SARS-CoV-2) emerging in Wuhan, China's Hubei province. The epidemic spread exponentially across the world and became a pandemic (1-3). Fever, dry cough, headache, and shortness of breath all typical symptoms, and can lead to death with acute respiratory distress syndrome. Diabetes mellitus, hypertension, coronary heart disease, chronic obstructive pulmonary disease and chronic kidney disease tend to be major risk factors for mortality (4). Real-time fluorescence reverse transcriptionpolymerase chain reaction (RT-PCR) is the preferred initial and reference diagnostic test for COVID-19. Chest computed tomography (CT) has gained importance due to the long result time and false negativity of the RT-PCR test $(5,6)$. Typical imaging findings for COVID-19 pneumonia are peripheral, bilateral, ground glass opacities with or without consolidation, and the differential diagnosis comprises influenza pneumonia, organizing pneumonia, drug toxicity, and connective tissue disease (7).

Due to immune dysfunction, steroid usage and immunotherapy, connective tissue diseases are vulnerable to viral infections. Few studies have identified the presence of concurrent COVID-19 in patients with connective tissue diseases $(8,9)$. In this article, we aimed to present the chest CT findings of a patient with connective tissue diseases who received immunosuppressed therapy.

\section{CASE REPORT}

A 28-year-old female patient applied to COVID-19 outpatient clinic with complaints of fever and weakness. The patient had a history of Sjogren's syndrome, mixed connective tissue disease and autoimmune hepatitis, so she was using methylprednisolone $12 \mathrm{mg} /$ 
day and hydroxychloroquine $200 \mathrm{mg} /$ day at the time of admission. The patient had a history of close contact with COVID-19 patient and her symptoms had just begun. Routine laboratory tests, nasopharyngeal sampling, and chest radiography were performed after the patient was examined. In the laboratory tests, the results were as follows: White blood cell (WBC) 8.800/ $\mathrm{mm} 3$, neutrophil count 6.600 , lymphocyte count 1.100 , hemoglobin $13.4 \mathrm{~g} / \mathrm{dl}$, platelet $357.000 / \mathrm{mm} 3$, C-reactive protein (CRP) $3.12 \mathrm{mg} / \mathrm{l}$, D-dimer $289 \mathrm{ng} /$ ml. Peripheral reticular opacities were seen on chest radiography, and chest CT scanning was performed due to suspicion. In chest $\mathrm{CT}$, there were basal predominantly, reticular and ground glass opacities that sparing the subpleural space in both lungs in favour of non-spesific interstitial pneumonia(NSIP) (Figure 1-2). In addition, round-shaped ground glass opacities were observed in the superior segment of the right lower lobe of the right lung (Figure 3). When the previous chest CT scans of the patient in our PACS system were examined, it was understood that the pattern of interstitial lung disease was similar, but the round-shaped ground glass opacity described in the lower lobe of the right lung was newly developed (Figure 2). These findings suggested COVID-19 pneumonia in addition to interstitial lung disease and were reported as indeterminate findings for COVID-19 according to the RSNA standard reporting language. Subsequently, the RT-PCR test confirmed COVID-19. The patient was hospitalized to arrange his treatment. Steroid therapy stopped, and favipiravir and hydroxychloroquine were prescribed in accordance with the recommendations of the Ministry of Health. In the following days, the patient's complaints regressed.

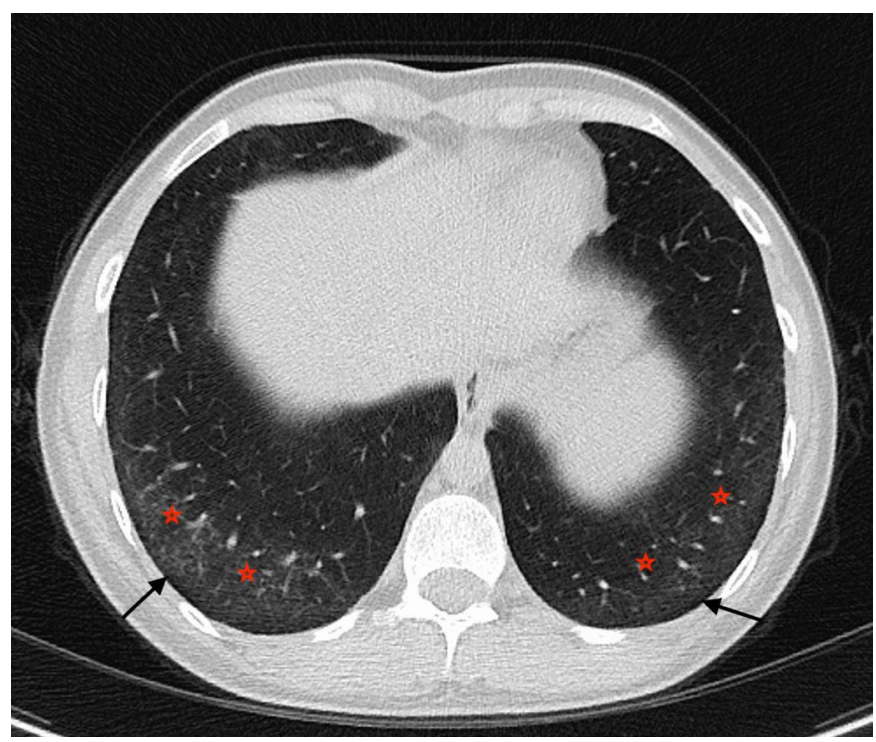

Figure 1. Basal reticular and ground glass opacities (red stars) with subpleural sparing (black arrows) are observed on axial chest CT image

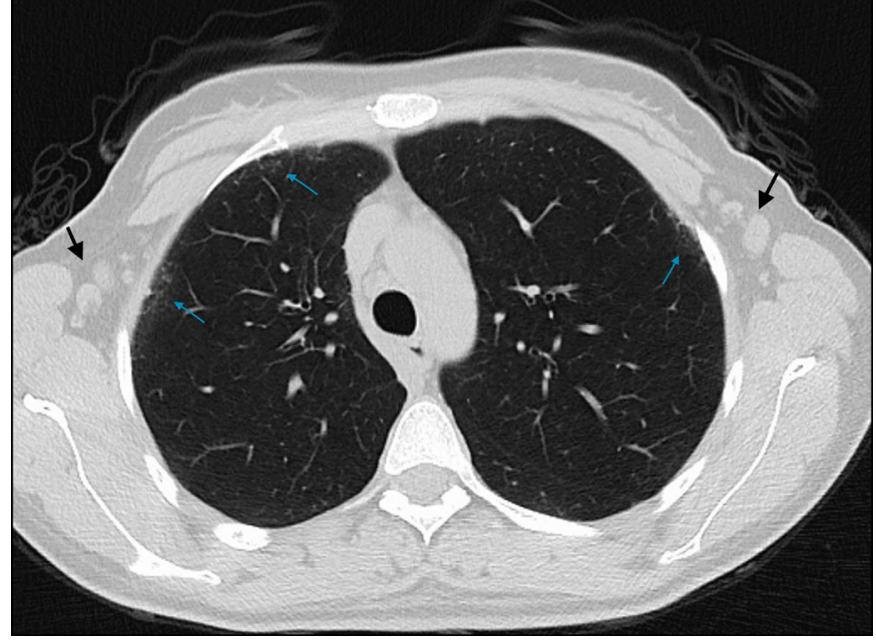

Figure 2. There is less reticulations in the anterior parts of the upper lobes (blue arrows) compared to the lower lobes. Axillary enlarged lymph nodes due to systemic inflammatory disease (black arrows) are observes on axial chest CT image

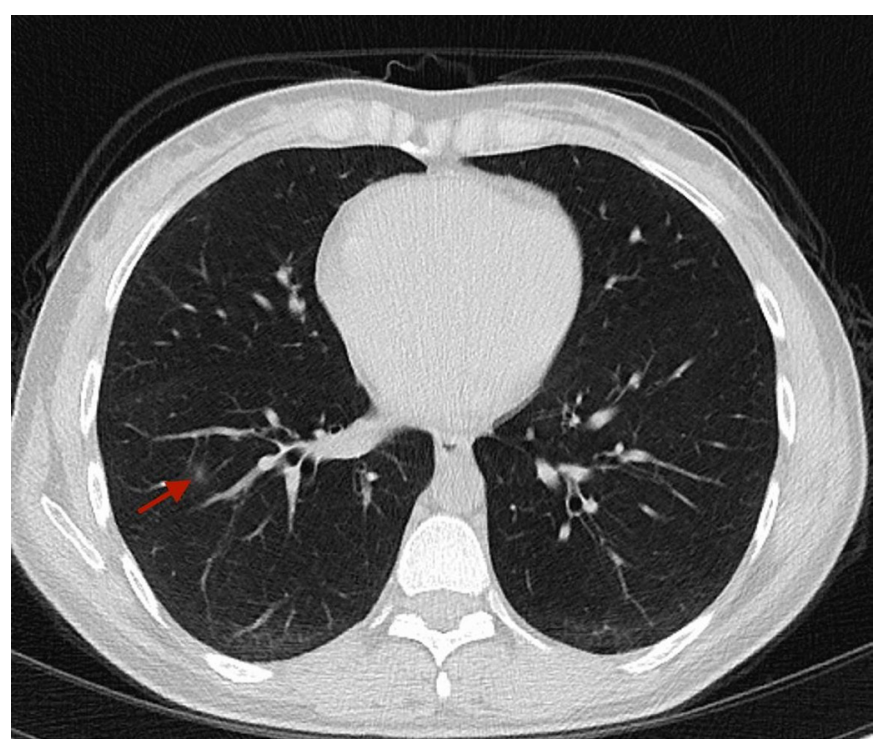

Figure 3. Round shaped ground glass opacity is observed in the lower lobe of the right lung on axial chest CT image (red arrow)

\section{DISCUSSION}

Since the onset of the new type of coronavirus pandemic, many publications have been published on the chest CT findings of the disease. Although it is not possible to say that viral pneumonic involvement is specific to coronavirus with its chest CT features, nevertheless, bilateral ground glass densities and consolidations are the most frequently reported chest CT involvement findings of the disease $(6,10)$. Normal chest CT findings cannot exclude the disease. Findings that can be observed as a single lesion in the early stages of the disease are manifested by infiltration areas, especially with peripherally located nodular or patchy ground glass opacity. As the disease gets more severe, the involvement becomes widespread and increases in density can be seen in the lesions. Interlobular and intralobular septal thickening may accompany. Respiratory failure may 
develop in the later stages of the disease. Some patients go from the early phase of the disease to the recovery period and heal without progress in the lesions (5,10-12). According to the RSNA consensus statement, connective tissue diseases are also included in the differential diagnosis of chest CT findings in COVID-19 (7). Patients with rheumatic immune diseases have immune dysfunction and are at a higher risk for new types of coronavirus infection than the normal population. Early diagnosis of the disease is even more important for these patients $(8,13)$. Ground glass opacity has been reported as the most common chest CT finding in patients with rheumatologic disease, as in those without the disease (14). In addition, pulmonary involvement findings of acute exacerbation periods of fibrotic interstitial pneumonias are considered in the differential diagnosis of COVID-19. Although new growth consolidation and ground glass opacity can be seen in both cases, peripherally located and round shaped ground glass opacity is more significant for COVID- 19 . Evaluation of the patient's history, exclusion of viral agents, and evaluation of the patient's previous imaging findings are important for differential diagnosis (15).

There were also known diagnoses of rheumatic and autoimmune diseases in our case. In addition to the findings of interstitial lung disease on chest CT findings, an area of round shaped ground-glass opacity located in the periphery, which is the most frequently reported finding of lung involvement of COVID-19 in the literature, was observed. This newly developed round shaped ground glass opacity can be distinguished from ground-glass opacities seen in interstitial lung diseases, especially NSIP. It was interpreted as suspicious in terms of COVID-19. The nucleic acid test performed confirmed the diagnosis of COVID-19.

\section{CONCLUSION}

Concomitant chronic diseases, especially diseases affecting the immune system, increase the risk for COVID-19 as in other infective diseases. In cases with a diagnosis of rheumatological disease, these diseases may have their own lung involvement. Additional COVID-19 involvement can be recognized together with the patient's history and chest CT findings. Early diagnosis of the disease can be lifesaver in these patients.

\section{ETHICAL DECLARATIONS}

Informed Consent: Written informed consent was obtained from all participants who participated in this study.

Referee Evaluation Process: Externally peer-reviewed.

Conflict of Interest Statement: The authors have no conflicts of interest to declare.
Financial Disclosure: The authors declared that this study has received no financial support.

Author Contributions: All of the authors declare that they have all participated in the design, execution, and analysis of the paper, and that they have approved the final version.

\section{REFERENCES}

1. Liu P, Tan XZ. 2019 Novel Coronavirus (2019-nCoV) Pneumonia. Radiology 2020; 295: 19.

2. Zhu N, Zhang D, Wang W, et al. A Novel Coronavirus from Patients with Pneumonia in China, 2019. N Engl J Med 2020; 382: 727-33. doi:10.1056/NEJMoa2001017.

3. Sun Z. Diagnostic Value of Chest CT in Coronavirus Disease 2019 (COVID-19). Current medical imaging 2020; 16: 274-5.

4. Ouédraogo D-D, Tiendrébéogo WJS, Kaboré F, Ntsiba H. COVID-19, chronic inflammatory rheumatic disease and antirheumatic treatments. Clinical Rheumatology 2020: 1.

5. Li M, Lei P, Zeng B, et al. Coronavirus disease (COVID-19): spectrum of CT findings and temporal progression of the disease. Acad Radiol 2020.

6. Li Y, Xia L. Coronavirus disease 2019 (COVID-19): role of chest CT in diagnosis and management. Am J Roentgenol 2020; 214: 1280-6.

7. Simpson S, Kay FU, Abbara S, et al. Radiological Society of North America Expert Consensus Statement on Reporting Chest CT Findings Related to COVID-19. Endorsed by the Society of Thoracic Radiology, the American College of Radiology, and RSNA. Radiology: Cardiothoracic Imaging 2020; 2: e200152.

8. Cheng C, Li C, Zhao T, et al. COVID-19 with rheumatic diseases: a report of 5 cases. Clinical rheumatology 2020: 1-5.

9. Santos CS, Morales CM, Álvarez ED, Castro CÁ, Robles AL, Sandoval TP. Determinants of COVID-19 disease severity in patients with underlying rheumatic disease. Clin Rheumatol 2020; 39: 2789-96.

10. Kanne JP. Chest CT Findings in 2019 Novel Coronavirus (2019$\mathrm{nCoV}$ ) Infections from Wuhan, China: Key Points for the Radiologist. Radiology 2020; 295: 16-7.

11. Pan Y, Guan H, Zhou S, et al. Initial CT findings and temporal changes in patients with the novel coronavirus pneumonia (2019$\mathrm{nCoV})$ : a study of 63 patients in Wuhan, China. Eur Radiol 2020: $1-4$.

12.Bernheim A, Mei X, Huang M, et al. Chest CT findings in coronavirus disease-19 (COVID-19): relationship to duration of infection. Radiology 2020: 200463.

13. Gianfrancesco M, Hyrich KL, Al-Adely S, et al. Characteristics associated with hospitalisation for COVID-19 in people with rheumatic disease: data from the COVID-19 Global Rheumatology Alliance physician-reported registry. Ann Rheum Dis 2020.

14. Ye C, Cai S, Shen G, et al. Clinical features of rheumatic patients infected with COVID-19 in Wuhan, China. Ann Rheum Dis 2020.

15.Larici AR, Cicchetti G, Marano R, et al. Multimodality imaging of COVID-19 pneumonia: from diagnosis to follow-up. A comprehensive review. Eur J Radiol 2020: 109217. 